\title{
Subcutaneous implantable cardioverter defibrillator in cardiomyopathies and channelopathies
}

Federico Migliore ${ }^{a}$, Francesco Pelliccia ${ }^{b}$, Camillo Autore ${ }^{c}$, Emanuele Bertaglia ${ }^{a}$, Franco Cecchi ${ }^{d}$, Antonio Curcio ${ }^{e}$, Luca Bontempi ${ }^{f}$, Antonio Curnis ${ }^{f}$, Paolo De Filippo ${ }^{g}$, Antonio D'Onofrio ${ }^{h}$, Pietro Francia ${ }^{c}$, Niccolò Maurizi', Beatrice Musumecic ${ }^{\mathrm{c}}$, Alessandro Proclemer ${ }^{j}$, Alessandro Zorzi ${ }^{\mathrm{a}}$ and Domenico Corrado ${ }^{\mathrm{a}}$

\begin{abstract}
Cardiomyopathies and channelopathies are heterogeneous disorders that increase the risk of sudden cardiac death (SCD). Implantable cardioverterdefibrillator (ICD) therapy is safe and effective for preventing SCD in patients at risk for malignant ventricular arrhythmias. Because of the poor positive predictive value of current risk stratification tools, the majority of patients implanted with an ICD will never receive a life-saving therapy but will be exposed to the risk of complications such as device infection, lead failure and inappropriate therapy. Subcutaneous ICD (S-ICD) now constitutes a valuable alternative to conventional transvenous ICD in patients with cardiomyopathies and channelopathies as it provides protection from SCD while avoiding the risks of intravascular lead infection or failure. This may be particularly advantageous for young patients with a very long life expectancy. On the other hand, S-ICD cannot deliver antitachycardia pacing or antibradycardia pacing. The purpose of this article is to review the available
\end{abstract}

\section{Introduction}

Sudden cardiac arrest is the most common cause of death in patients with cardiomyopathies and channelopathies. ${ }^{1}$ For this reason the implantable cardioverter defibrillator (ICD) has become the gold standard for prevention of sudden cardiac death (SCD). ${ }^{2}$ The advent of the fully subcutaneous ICD (S-ICD) has provided a valuable alternative for patients with cardiomyopathies and channelopathies. ${ }^{3}$ The S-ICD allows patients who do not need pacing to benefit from arrhythmia protection without the risk of systemic infection and potentially reducing lead failure, which is an important complication of transvenous ICD (T-ICD). ${ }^{4}$ This may be particularly advantageous for young patients with a long life expectancy who might avoid the complications associated with transvenous leads as well as the procedural risks associated with their extraction. ${ }^{5}$

This review article focuses on the available scientific information on safety and efficacy of the use of S-ICD in patients with cardiomyopathies and channelopathies in evidence and the future perspectives of S-ICD therapy in patients with cardiomyopathies or channelopathies.

J Cardiovasc Med 2018, 19:633-642

Keywords: cardiomyopathies, channelopathies, subcutaneous implantable cardioverter defibrillator, sudden cardiac death

${ }^{a}$ Department of Cardiac, Thoracic and Vascular Sciences, University of Padova ${ }^{b}$ Department Attilio Reale, Sapienza University, ${ }^{\mathrm{C}}$ Department of Clinical and Molecular Medicine, Sapienza University of Rome, Rome, ${ }^{\mathrm{d}}$ Department of Clinical and Experimental Medicine, University of Florence, Florence, e Division of Cardiology, Department of Medical and Surgical Sciences, University Magna Graecia, Campus di Germaneto, Catanzaro, 'Division of Cardiology, Spedali Civil Hospital, Brescia, ${ }^{9}$ Cardiac Electrophysiology and Pacing Unit, Cardiovascular Department, Ospedale Papa Giovanni XXIII, Bergamo, hDepartment of Cardiology, Monaldi Hospital, AORN Ospedali dei Colli, Naples,

'Cardiomyopathy Unit, Careggi University Hospital, Florence and 'University Hospital Santa Maria della Misericordia, Udine, Italy

Correspondence to Federico Migliore, MD, PhD, FAIAC, FESC, FEHRA Department of Cardiac, Thoracic and Vascular Sciences, University of Padua Medical School, Via N. Giustiniani 235121 Padova, Italy.

Tel: +39049 8212332; fax: +390498212309;

e-mail: federico.migliore@libero.it

Received 30 April 2018 Accepted 12 August 2018

order to increase knowledge for the optimal use of this innovative technology in this specific group of patients.

\section{Prevention of sudden cardiac death in cardiomyopathies and channelopathies}

Risk stratification in order to identify patients at high risk of malignant ventricular arrhythmias at the time of initial evaluation and during follow-up is strongly recommended by current guidelines. ${ }^{7}$ However, weighting the risks and benefits of ICD therapy in the daily clinical practice remains a difficult task and the decision to implant such a device has to take into account the needs and desires of the individual patient.

\section{Complications of conventional implantable cardioverter defibrillator}

Transvenous ICDs are implanted worldwide in an increasing number of patients. ${ }^{2}$ Unfortunately, complications or failure of a conventional ICD lead may result in adverse clinical events, including failure to pace and 
defibrillate, inappropriate shocks, pocket infection, endocarditis, cardiac perforation and even death. ${ }^{5}$ Recent studies have reported an increased risk of re-intervention after implantation of high-voltage ICD leads compared with right ventricular pacing leads. ${ }^{6}$ This can be attributed to the more complex structure, larger caliber, and increased rigidity of high-voltage leads which, in addition, require to satisfy more stringent lead parameters at the time of implant and during follow-up.

The risk of complications associated with T-ICD implantation ranges between 3 and $8 \%$. Kirkfeldt et al. ${ }^{8}$ reported that $9.5 \%$ of patients who received an ICD experienced at least one complication, whereas $0.6 \%$ of patients had multiple complications. Lead-related re-intervention was the single most common complication (2.4\%). System upgrades or lead revisions are associated with a higher rate of complications than first implants, primarily because of the higher risk of infections that are infrequent but carry significant morbidity and mortality. Tarakji $e t a l .{ }^{9}$ aimed to assess risk factors for 1-year mortality among patients with ICD infection and to evaluate if the type of infection and the presence of vegetation affect survival. The authors reported a $20 \%$ mortality at 1 -year of follow-up; 'endovascular infection' was associated with significantly higher 1year mortality compared with 'pocket infection' independently of the presence of vegetations. Olde Nordkamp et al. ${ }^{10}$ performed a systematic review and meta-analysis of inappropriate shocks and/or other ICD-related complication rates in patients with inherited arrhythmia syndromes. In this population, inappropriate shocks occurred in $20 \%$ of patients with an annual rate of $4.7 \%$ per year. Moreover, $22 \%$ experienced ICD-related complications $(4.4 \%$ per year) and ICD-related mortality was $0.5 \%$. Lead malfunction was the primary complication in this study occurring in $10 \%$ of patients.

Inappropriate shocks and device-related complications may be more prevalent in young patients because of an active lifestyle and because of a longer period of exposition to the risks of ICD therapy. Migliore $e t$ al. ${ }^{11}$ specifically evaluated the risk-benefit ratio of ICD in 96 young patients with cardiomyopathies and channelopathies who were 18-35 years old at the time of ICD implantation. During a mean follow-up of $72.6 \pm 53.3$ months, 20 patients $(21 \%)$ experienced at least one appropriate ICD intervention (4\%/year), $9(9 \%)$ at least one inappropriate shock and $20(21 \%)$ suffered from device-related complications requiring system revision. In particular, the most common complication, which occurred in 9 patients, was lead failure/fracture requiring lead extraction. ${ }^{11}$

\section{Potential advantages of subcutaneous implantable cardioverter defibrillator}

The S-ICD might overcome the limitations of conventional ICDs, as the absence of transvenous leads might reduce implantation and lead-related complications in patients without an indication for brady and antitachycardia pacing (ATP). The EFFORTLESS registry $^{12}$ has provided data to evaluate the safety and efficacy of the S-ICD in a large population.

Patients were followed-up for $3.1 \pm 1.5$ years and 82 completed a 5-year follow-up period. The S-ICD system and procedure complication rate were $4.1 \%$ at 30 days and $8.4 \%$ at 360 days. The 1 -year complication rate trended toward improvement from the first $(11.3 \%)$ to the last (7.4\%) quartile of enrollment. Few device extractions occurred because of need for antitachycardia $(n=5)$, biventricular $(n=4)$ or bradycardia pacing $(n=1)$. Inappropriate shocks occurred in $8.1 \%$ of patients at 1 year and $11.7 \%$ after 3.1 years. The 1 -year and 5 -year rates of appropriate shock were 5.8 and $13.5 \%$, respectively. Conversion success for discrete spontaneous episodes was $97.4 \%$ overall. Overall, these data suggest that SICD is associated with favorable outcomes.

\section{Subcutaneous implantable cardioverter defibrillator: device and implantation technique}

The S-ICD consists of a pulse generator and a single lead with a shock coil. The pulse generator is implanted in the left lateral position. A single lead for sensing is usually positioned $1-2 \mathrm{~cm}$ to the left of and parallel to the sternum, with the distal tip near the manubriosternal junction. ${ }^{4}$ The lead consists of sensing electrodes at the subxiphoid (proximal) and manubriosternal junction (distal) positions, separated by an $8-\mathrm{cm}$ shocking coil (Fig. 1). The conventional implantation technique requires three incisions to implant the electrode and

Fig. 1

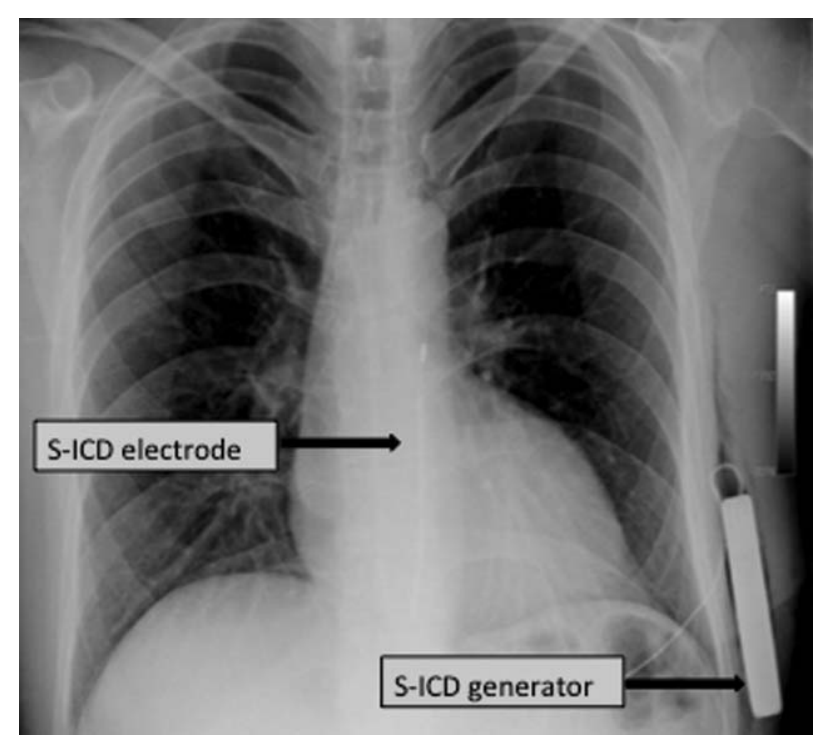

Chest X-ray in a patient with subcutaneous implantable cardioverterdefibrillator. The generator is positioned on the lateral side of the chest wall. The defibrillator lead is tunnelled under the skin, and located along the chest wall in the left parasternal position. 
the device that is positioned in a subcutaneous pocket at the level of the left axillary line. ${ }^{4}$

Recently, a two-incision technique, eliminating the superior parasternal incision, has been developed. ${ }^{13}$ This lessinvasive and simplified technique is safe and may help reducing complications in S-ICD patients. An intermuscular pocket has also been proposed as an alternative to the standard subcutaneous pocket. This technique consists of creating a pocket for the device between the anterior surface of the serratus anterior muscle and the posterior surface of the latissimus dorsi muscle using anatomical landmarks. ${ }^{14-16}$ The intermuscular technique provides a wider pocket in a more posterior left axillary region, an extra layering, and a virtual space between device and chest, resulting in potential reduction of pocket-related complications, especially skin erosions and infections. ${ }^{16,17}$ As the whole system, including the electrode, is located outside the vascular system, S-ICD is by far less prone to infection, and the lead is less prone to fracture. Due to the lack of an electrode, however, the device is unable to deliver bradycardia pacing, ATP, or cardiac resynchronization therapy. On the other hand, the fact that the device is not in contact with the heart allows patients with S-ICD to safely undergo magnetic resonance imaging. ${ }^{5}$

\section{Indications to subcutaneous implantable cardioverter defibrillator in patients with cardiomyopathies and channelopathies}

The S-ICD is approved for the prevention of SCD among candidates for a conventional T-ICD but who do not have an indication for antibradycardia pacing or cardiac resynchronization therapy, recurrent ventricular tachycardia (VT) treatable with ATP, or preexisting unipolar pacemaker leads. Although patients enrolled in early S-ICD prospective registries were relatively young, with less advanced heart disease, and sometimes with 'niche' indications (i.e. high risk of infections, congenital heart disease, poor vascular access), contemporary S-ICD patients are more severely diseased and have more comorbidities. ${ }^{18}$ Notwithstanding, these young patients with cardiomyopathies and primary electrical heart diseases are ideal candidates for ICD (Table 1). Indeed, cumulative lead-related and device-related complications of the T-ICD are well known and have been reported to be as high as $2-4 \%$ per year in patients with cardiomyopathies. ${ }^{10,11}$ The S-ICD is safe and effective in cardiomyopathy patients, and because of the negligible risk of systemic infection and increased durability of the lead system, it is an attractive option for most cardiomyopathy patients without a pacing indication. ${ }^{19}$

At present, S-ICDs is recommended only in a specific subset of patients. The 2015 guidelines for the prevention of SCD of the European Society of Cardiology have established a Class IIa-level C recommendation for the use of S-ICD as an alternative to transvenous ICDs when pacing therapy for bradycardia, cardiac resynchronization or ATP is not needed. ${ }^{20}$ Also, S-ICD has a Class IIb-level $\mathrm{C}$ recommendation as an alternative to T-ICD in case of difficult venous access, young patients, and after the removal of a previous T-ICD system for infection.

Table 1 Number of patients with cardiomyopathies or channelopathies with subcutaneous implantable cardioverter defibrillator included on S-ICD registries

\begin{tabular}{|c|c|c|}
\hline Author group & Reference & Number of patients \\
\hline \multicolumn{3}{|l|}{ Hypertrophic cardiomyopathy } \\
\hline Arias AM, et al. & Rev Esp Cardiol 2017 Dec 5. pii: S1885-5857 & 9 \\
\hline Özkartal T, et al. & Swiss Med Wkly 2017;147:w14518 & 9 \\
\hline Maurizi $\mathrm{N}$, et al. & Int J Cardiol 2017; 231:115-119 & 50 \\
\hline Lambiase PD & Heart Rhythm 2016; 13:1066-1074 & 99 \\
\hline Frommeyer G, et al. & Clin Res Cardio/ 2016; 105:89-93 & 18 \\
\hline Francia $P$, et al. & J Cardiovasc Electrophysiol 2015; 26:893-899 & 3 \\
\hline Galvao $P$, et al. & Rev Port Cardiol 2014; 33:511-517 & 4 \\
\hline Kobe J, et al. & Heart Rhythm 2013; 10:29-36 & 10 \\
\hline \multicolumn{3}{|l|}{ Dilated cardiomyopathy } \\
\hline Kobe J, et al. & Heart Rhythm 2013; 10: 29-36 & 69 \\
\hline Brouwer TF, et al. & J Am Coll Cardiol 2016; 19: 2047-2055 & 30 \\
\hline \multicolumn{3}{|c|}{ Arrhythmogenic cardiomyopathy } \\
\hline Migliore $\mathrm{F}$, et al. & JACC Clinical Electrophysiol 2017; 3:785-786 & 1 \\
\hline Boersma l, et al. & $J A C C 2017 ; 70: 830.841[12]$ & 32 \\
\hline \multicolumn{3}{|l|}{ Brugada syndrome } \\
\hline Kamakura $\mathrm{T}$, et al. & Heart Vessels $2017 ; 32: 1277-1283$ & 6 \\
\hline Ishibashi K, et al. & J Arrhythm 2017; 33:156-158 & 1 \\
\hline Morani G, et al. & Pacing Clin Electrophysiol 2017; 40:1180-1183 & 1 \\
\hline Sideris S, et al. & Hellenic J Cardiol 2017; 58:4-16 & 2 \\
\hline Hai JJ, et al. & Europace 2015; 17 Suppl 2:ii63-8 & 6 \\
\hline Yap SC, et al. & Heart Rhythm Case Rep 2015;1:156-158 & 1 \\
\hline Galvão $P$, et al. & Rev Port Cardiol 2014; 33:511-517 & 4 \\
\hline Köbe J, et al. & Heart Rhythm 2013;10:29-36 & 6 \\
\hline Olde Nordkamp LR, et al. & J Am Coll Cardiol 2012; 60:1933-1999 & 9 \\
\hline Jarman JW, et al. & Eur Heart J 2012; 33:1351-1359 & 3 \\
\hline De Maria E, et al. & J Interv Card Electrophysiol 2012;34:105-113 & 1 \\
\hline Dabiri Abkenari L, et al. & Clin Res Cardiol 2011; 100:737-744 & 2 \\
\hline Conte G, et al. & Europace $2018 ; 20: 1188-1193$ & 61 \\
\hline
\end{tabular}


The 2017 American guidelines on prevention of $\mathrm{SCD}^{7}$ state that S-ICD is recommended in patients who meet criteria for an ICD who have inadequate vascular access or are at high risk for infection, and in whom pacing for bradycardia or VT termination or as part of cardiac resynchronization therapy is neither needed nor anticipated (Class I). Moreover, S-ICD is an option in patients who meet indication for an ICD if pacing for bradycardia or VT termination or as part of cardiac resynchronization therapy is neither needed nor anticipated (class II). Finally, S-ICD is contraindicated if bradycardia pacing, cardiac resynchronization therapy or ATP is required (class III).

\section{Subcutaneous implantable cardioverter defibrillator in hypertrophic cardiomyopathy}

Hypertrophic cardiomyopathy (HCM) is a genetic sarcomeric disorder associated with myocyte disarray and scar deposition, which predisposes to malignant ventricular arrhythmias. ${ }^{1}$ The disease is a leading cause of SCD in young adults ${ }^{21}$ and high-risk HCM patients benefit from ICD therapy, although they may suffer long-term devicerelated complications. ${ }^{21}$ The S-ICD is a promising option for HCM patients, who are usually young and rarely require pacing. ${ }^{22-24}$

The S-ICD performance in HCM patients has been evaluated extensively. ${ }^{23-28}$ It proved effective in the detection and cardioversion of induced VT/ventricular fibrillation (VF) with equivalent frequency of inappropriate shocks between HCM and non-HCM patients. Specifically, reported rates of induced VT/VF conversion were $95.5-98 \%$ at $65 \mathrm{~J}$ and $98-100 \%$ at $80 \mathrm{~J} .{ }^{26-28}$ Of note, defibrillation failures at $65 \mathrm{~J}$ were mostly attributed to severe obesity and its consequences on defibrillation threshold rather than on the HCM phenotype. ${ }^{26,27}$ In fact, patients with left ventricular maximal wall thickness at least $30 \mathrm{~mm}$ or a marked increase of left ventricular mass showed a similar rate of effective $65 \mathrm{~J}$ defibrillation of induced VT/VF to those wild milder phenotype. ${ }^{26-29}$ New implantation techniques, including intermuscular placement, have been developed in order to avoid this problem. ${ }^{14-16}$

Reduction of inappropriate shocks remains a significant challenge. Up to $12.5 \%$ of patients with HCM experienced inappropriate shocks during a median follow-up of 2 years, mainly due to T-wave oversensing. ${ }^{28}$ However, recent improvements in the S-ICD discrimination algorithm as well as accurate patient selection are offering a partial mitigation to such an undesired event. Pocket infection occurred in up to $5 \%$ of patients, but they were all managed with standard antibiotic treatment. ${ }^{28}$ Lead fracture was extremely rare as well as lead displacement. ${ }^{28}$ In conclusion, in HCM patients who do not need pacing, S-ICD is a safe and effective prophylactic therapy for SCD prevention ${ }^{23-28}$ (Fig. 2).
The risk of inappropriate shocks should be mitigated by accurate preimplant evaluation and dual zone programming. Specifically, because HCM patients often show markedly abnormal ECG, a careful preimplantation evaluation is required to avoid inappropriate sensing by the $\mathrm{S}$ ICD, which uses a morphology-based rhythm discrimination algorithm. ${ }^{23-25}$ Recent studies examining ECG vector screening at rest have demonstrated that $7-16 \%$ of HCM patients fail preimplant screening, mainly because of massive hypertrophy, broad QRS, prior myectomy procedure, and $\mathrm{T}$-wave inversions in more than two leads on standard 12-lead electrocardiogram. ${ }^{24,25}$ Moreover, in HCM, the S-ICD system may be challenged in the differentiation between supraventricular tachycardia and V'T because of the relative R:T wave amplitude variation during tachycardia, potential ST-segment elevation, or development of rate-dependent aberrancy. The latter can be mitigated by storing a template of previous aberrancy (i.e. during exercise), altering the sensing vector or modifying the cut-off rate, but cannot be avoided if the changes are transient or unpredictable. ${ }^{28}$ These dynamic QRS-T wave changes emphasize the importance of exercise testing these patients prior to implant to optimize template vector selection. ${ }^{23-28}$ Usually two (of the three available) vectors should pass the screening process in order to provide alternative vectors for sensing. However, a single passing vector that remains eligible both at rest and during exercise is acceptable when avoiding endovascular leads is warranted. Also, right parasternal chest electrodes should be assessed in the case of screening failure using conventionally positioned electrodes. ${ }^{24}$

\section{Subcutaneous implantable cardioverter defibrillator in dilated cardiomyopathy}

In the context of dilated cardiomyopathy (DCM), ICD is recommended in patients with haemodynamically not tolerated VT/VF or in patients with symptomatic heart failure and an ejection fraction less than $35 \%$ despite at least 3 months of optimal medical therapy. ${ }^{7}$ Most DCM patients with ICD indication are suitable S-ICD recipients, ${ }^{3}$ but, in clinical practice, only a few centers propose S-ICD as a first-choice therapy. ${ }^{29}$

The absence of ATP functionality is an important barrier to the widespread adoption of the S-ICD in patients with DCM. The device can obviously not be used in patients requiring bradycardia or resynchronization pacing. However, the need for pacing in DCM patients in historical ICD trials ${ }^{30}$ and in recent S-ICD studies ${ }^{12,31}$ has been very low when patients are properly selected and there is no indication in the current guidelines for preventive pacemakers when conduction system disease is not expected. Similarly, the role of ATP in the era of therapy reduction programming is not clear. In the MADIT-RIT trial, ${ }^{32}$ very few patients received ATP in the high-rate and delayed-therapy arms. These findings raise the 
Fig. 2

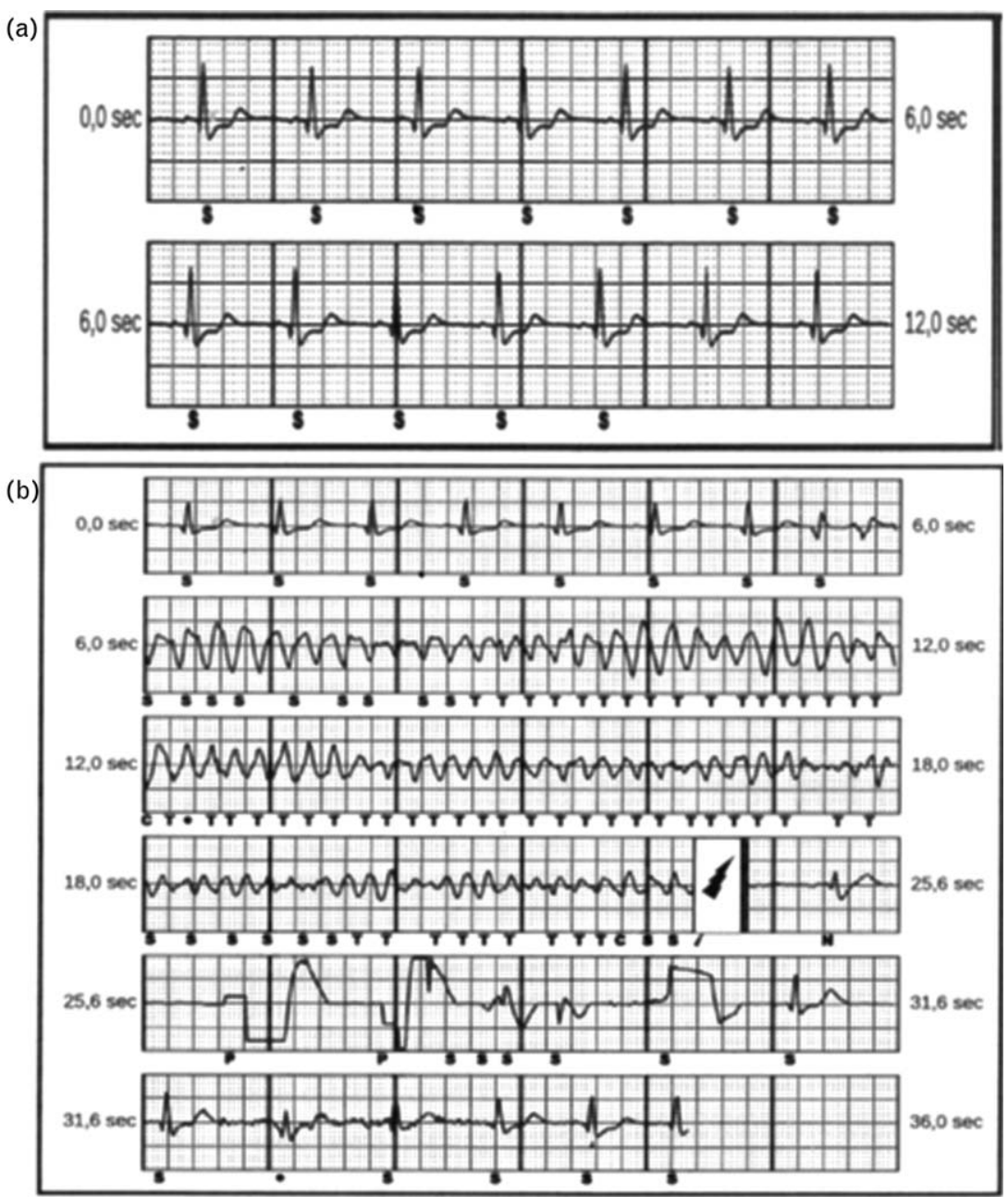

A 31-year-old man with hypertrophic cardiomyopathy (left ventricular maximal wall thickness $38 \mathrm{~mm}$ ) who underwent subcutaneous implantable cardioverter defibrillator implantation for primary prevention (a). Two years after follow-up, the patient received appropriate ICD intervention on ventricular fibrillation (b). ICD, implantable cardioverter defibrillator.

question of whether the potential benefit of ATP is outweighed by the risk of transvenous lead complications.

Although no study has prospectively investigated the benefit of S-ICD in specific etiological subgroups of DCM, patients with this cardiomyopathy are well represented in the trials. Particularly, several clinical studies have demonstrated the safety and effectiveness of the $S$ ICD in these patients. ${ }^{12,31}$ In a propensity-matched cohort (20\% of patients with DCM) of S-ICD, the authors demonstrated that the S-ICD has a significant benefit over ICDs with respect to lead-related complications. ${ }^{33}$ More recently, the EFFORTLESS S-ICD Study Midterm Results ${ }^{12}$ showed that the rate of complications directly caused by the S-ICD system as well as the rate of all S-ICD system and procedure-related complications were low. Specifically, no significant higher risk of complications was observed in DCM patients. Moreover, the EFFORTLESS/IDE pooled cohort confirmed that the favorable outcomes achieved with the S-ICD continue up to 3 years postimplantation. ${ }^{12}$ In the coming years, the UNTOUCHED (Understanding Outcomes with the S-ICD in Primary Prevention Patients with Low Ejection Fraction Study), a prospective registry of primary prevention patients with reduced left ventricular ejection fraction ${ }^{34}$ will provide valuable data on S-ICD in this cohort of patients.

\section{Subcutaneous implantable cardioverter defibrillator in arrhythmogenic cardiomyopathy}

According to the 'International Task Force Recommendations for arrhythmogenic right ventricular cardiomyopathy (ARVC) treatment, ${ }^{35}$ the decision whether to implant a S-ICD in ARVC needs to be patient-specific, 
balancing lead-related complications with the likelihood of recurrent VT that may be effectively pace-terminated. Antitachycardia pacing appears very effective in terminating VT in ARVC patients. Among 108 patients with an ICD (mean age 40 years, $48 \%$ secondary prevention) enrolled in the North American ARVC registry, ${ }^{36} 48$ experienced a total of 489 episodes of monomorphic VT during follow-up: of those, 450 were treated with ATP that terminated the arrhythmia in $92 \%$ of cases. The efficacy of ATP was consistent throughout different tachycardia cycle length suggesting that ATP can interrupt even fast VT. These data suggest that the inability of the S-ICD to deliver ATP is an important limitation in the setting of ARVC, especially considering that a subanalysis of the MADIT trial showed that ICD shocks, but not ATP, were associated with adverse prognosis in ICD recipients. ${ }^{32}$ However, to judge whether this should be regarded as an absolute contraindication to S-ICD in ARVC, other factors should be taken into account. First of all, it has to be noted that $\approx 2 / 3$ of V'Ts that were interrupted by ATP in the North American study were slower than 200 beats per minute, that is, probably nonlife-threatening considering that the majority of ARVG patients have a normal or near-normal left-ventricular ejection fraction. ${ }^{36}$ Moreover, the arrhythmic presentation (i.e. VF vs. VT) of ARVC is age-dependent: although older patients with advanced disease more often experience re-entrant VT around a stable fibro-fatty myocardial scar, in young patients it is common to observe the abrupt onset of VF reflecting acute electrical instability of early disease, which progresses through socalled 'hot phases' (recurrent bouts of acute myocyte death with reactive inflammation). ${ }^{37,38}$ Finally, young patients are particularly prone to lead-related complications requiring device explant. ${ }^{11}$ Taking all these factors into account, S-ICD may be a valuable alternative to TICD in young ARVC patients without a history of sustained monomorphic VT.

In the assessment of S-ICD eligibility of ARVC patients, some peculiar aspects of the disease should be taken into account. First, patients typically exhibit reduced voltages of QRS complexes and large, negative $\mathrm{T}$ waves and/or right atrial enlargement (peaked $\mathrm{P}$ waves) that may cause double-counting and inappropriate interventions. Second, repolarization abnormalities may significantly modify with increasing heart rate so that electrocardiographic screening should be performed both at baseline and during exercise testing (Fig. 3). ${ }^{39-41}$

\section{Subcutaneous implantable cardioverter defibrillator in Brugada syndrome}

Brugada syndrome $(\mathrm{BrS})$ is an inherited arrhythmogenic disorder that exposes young men to polymorphic VT or $\mathrm{VF}$ and SCD. ${ }^{42-48}$ To date, the literature focusing on S$\mathrm{ICD}$ in $\mathrm{BrS}$ is limited. The main limitation is represented by lack of pacing capability. Patients with $\mathrm{BrS}$ are potentially at risk of progressive conduction disorders because of genetically defective sodium channels similar to the Lenègre disease phenotype. ${ }^{47}$ However, recent reports underlined that advanced atrioventricular block only develops at advanced age, whereas ATP is not considered essential, as VF and polymorphic V'T are the most common arrhythmias occurring in $\mathrm{BrS}^{44}$ while monomorphic VT is rare. ${ }^{49}$

Similarly to other cardiomyopathies, reduction of inappropriate shocks is pivotal in patients with $\mathrm{BrS}$. In this regard, it is important to accurately assess the sensing vectors at preimplant screening, as dynamic ST-segment elevation in right precordial leads is observed in various daily life situations such as during sleep, following a meal, and after exercise ${ }^{50}$ (Fig. 4). Screening tests conducted only at rest may be inadequate for the prediction of the $\mathrm{T}$ wave oversensing in S-ICD candidates with $\mathrm{BrS}$, and drug challenge test may be useful in appropriately qualifying BrS patients for S-ICD. ${ }^{51}$ To increase the precision of arrhythmia detection by S-ICD, an updated software applies high-pass filter that diminishes the amplitude of lower frequency signals such as $\mathrm{T}$ waves, whereas higher frequency signals such as $\mathrm{R}$ waves, polymorphic VT and VF amplitudes remain largely unchanged. ${ }^{52}$ Finally, supraventricular tachycardias, including atrial fibrillation, are synergistically discriminated by two algorithms named ventricular scatter analysis, which monitors atrial fibrillation vs. nonatrial fibrillation episodes based on R-R intervals, and heart rate density index algorithm, which controls heart rate distribution. ${ }^{53}$

\section{Potential limitation of the subcutaneous implantable cardioverter defibrillator}

Selection of eligible patients is crucial for S-ICD and is based on a template ECG morphology screening, which evaluates QRS complex and T-wave morphology in order to detect QRS/T-wave oversensing. ${ }^{54}$ Inability to find an ECG lead suitable for the screening template implies a potential high risk of inappropriate detections by S-ICD owing to oversensing of cardiac signals, mostly $\mathrm{T}$ wave, and advises against S-ICD implant. ${ }^{54}$ Predictors of ECGscreening failure, especially in younger patients, ${ }^{55}$ are longer QRS duration, longer QTc interval, and a lower $\mathrm{R}$ to $\mathrm{T}$ amplitudes (R:T ratio), ${ }^{56-58}$ which are depolarization/repolarization ECG abnormalities typically observed in patients with cardiomyopathies and channelopathies. $^{21,37,44}$ These peculiarities can lead to a higher rate of ECG-screening failure in this subgroup of patients, decreasing the number of those who could benefit from the attractive therapeutic option of S-ICD implantation. In this regard, Olde Nordkamp et al. ${ }^{56,57}$ showed that HCM was an independent predictor of screening failure, but this was not reported in other studies. ${ }^{23,24,55}$ Conte et al. ${ }^{59}$ addressed rate and specific aspects of S-ICD eligibility in patients with inherited primary arrhythmia syndromes. They found that $13 \%$ of patients potentially 
Fig. 3

(a)

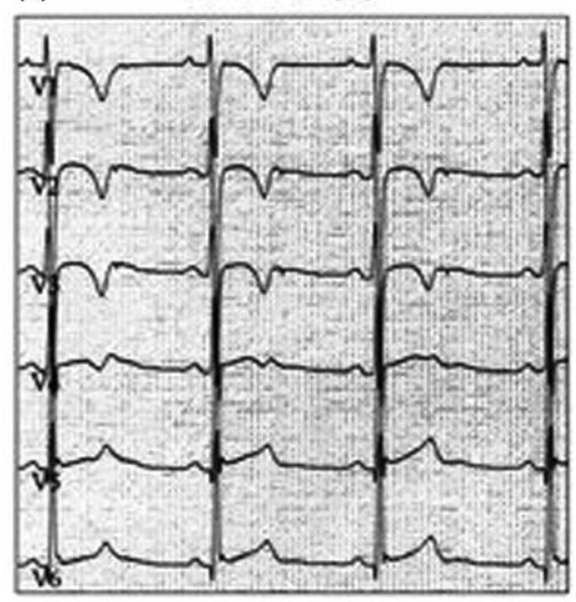

(c) ECG during stress test

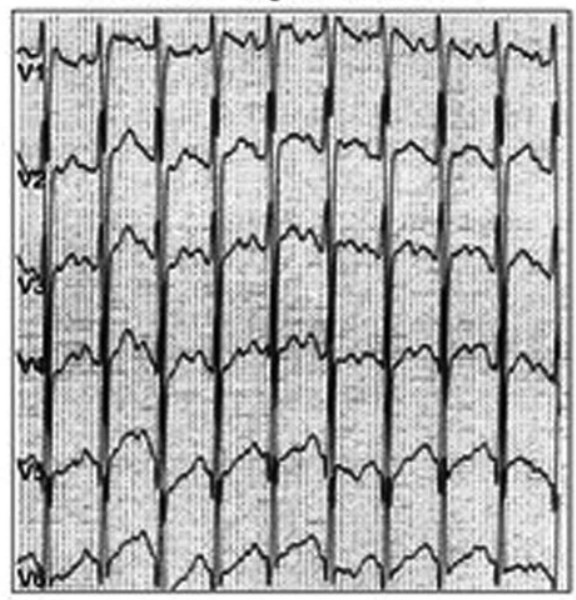

(b)
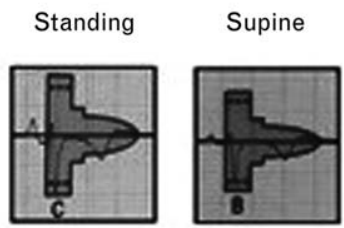

NO

I
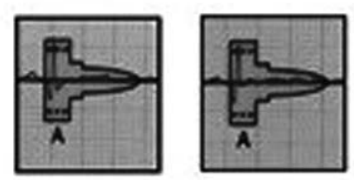

YES
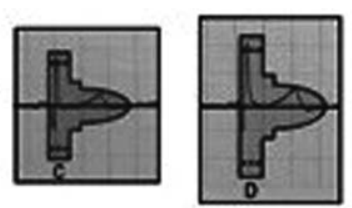

\section{YES}

(d)
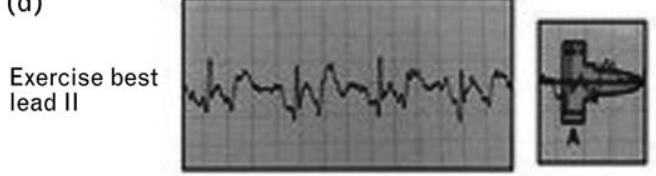

NO

Exercise best lead III

NO
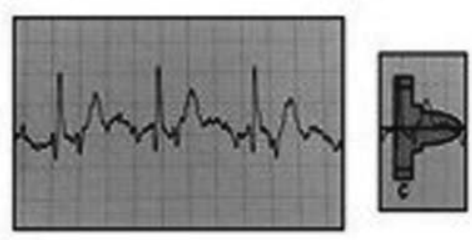

Representative example of a young patient affected by arrhythmogenic cardiomyopathy with right precordial negative T waves on basal ECG (a) that normalize during exercise (c). Morphology analysis of QRS and T wave showed that although two of three sense vectors were appropriate at baseline (b), they became inappropriate during exercise (d), precluding a subcutaneous defibrillator implantation. Reproduced from Migliore et al. ${ }^{40}$

candidates for S-ICD implantation, failed the screening morphology analysis. In particular, patients with $\mathrm{BrS}$ showed the highest rate of screening failure and the lowest number of appropriate S-ICD vectors compared with patients with other channelopathies. ${ }^{59}$ However, in another study, Rudic et al..$^{60}$ did not observe any screening failure in a cohort of patients with inherited primary arrhythmia syndrome. The different samples size and the ECG screening performed or not after ajmaline challenge may explain the discrepancy in eligibility rates.

In the assessment of S-ICD eligibility of patients with cardiomyopathy and channelopathies, some peculiar aspects of the diseases should be taken into account. Important dynamic changes in surface ECG may occur with increasing heart rate, so that ECG screening should be considered both at baseline and during exercise stress testing. ${ }^{24,40,61}$ Moreover, cardiomyopathies are progressive diseases predisposing this population to worsening of sensing reliability leading to possible cardiac and/or noncardiac oversensing and subsequent inappropriate therapy, increasing defibrillator energy or pacing requirement. ${ }^{37,62}$ Consequently, it may be desirable to have at least two of three vectors suitable in S-ICD screening template in order to facilitate future management of potential device-related oversensing during follow-up.

Possible strategies that may increase S-ICD eligibility and reduce inappropriate shocks are proper 
(a)

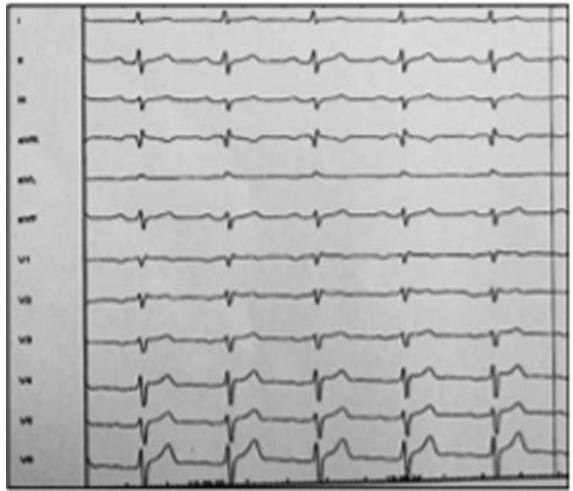

(b)

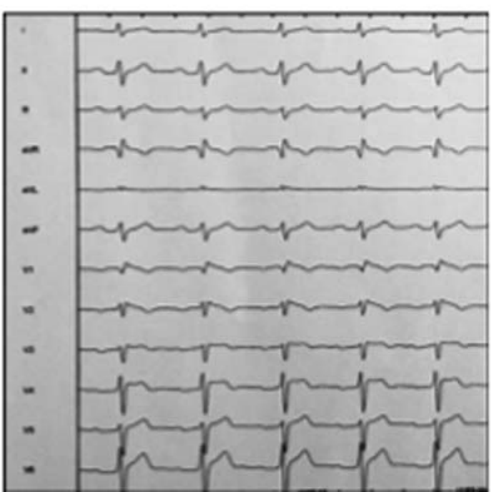

(c)

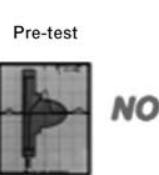

(d)

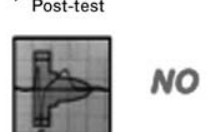

"

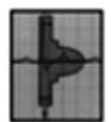

YES

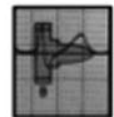

NO

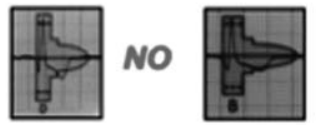

A representative example of preimplant screening test in a patient wit Brugada syndrome. Normal QRS, ST-segment and T wave on basal ECG (a) and 'coved-type' ST-segment elevation (type 1 Brugada ECG) after ajmaline test (b). Morphology analysis of QRS and T wave at baseline showed that only one (II) of the three sense vectors was appropriate because of the high amplitude of the T wave of the sense vectors I and III (c). Morphology analysis repeated after ajmaline administration showed that also the previously appropriate sense vector was no longer acceptable because of the occurrence of the 'coved-type' ST-T waveform (d). Owing to the lack of consistency of appropriate sense vectors both at baseline and after ajmaline challenge, the patient received a transvenous single-chamber ICD. ICD, implantable cardioverter defibrillator.

preimplantation ECG screening, ${ }^{24,40,61}$ device programming (single-zone vs. dual-zone programing), ${ }^{12}$ new implantation techniques, ${ }^{13-16}$ and software upgrading including the 'SMAR'T Pass', which is a recently introduced filter that has been reported to reduce over sensing. ${ }^{63}$

The major limitation for S-ICD comes from the lack of permanent pacing capability and inability to deliver ATP, which may be an effective 'pain-free' therapy in patients with structural heart diseases including nonischemic cardiomyopathies. ${ }^{7,32,36}$ Patients who undergo ICD implantation because of clinical episodes of monomorphic VT should receive a device able to deliver ATP in order to avoid shocks; hence, for these patients S-ICD should not be the first choice. Notably, in the long-term follow-up of the entire EFFORTLESS registry cohort, only $0.5 \%$ of patients had the device removed for conceived need for ATP. ${ }^{12}$ These data suggest that in patients without a history of sustained VT implantation of a S-ICD should not be excluded based only on the inability of the device to deliver ATP. Instead, S-ICD may be a valuable alternative to T-ICD in patients with cardiomyopathies and channelopathies and the potential risk of S-ICD shocks rather than ATP on future monomorphic VT must be weighed against the high incidence of long-term leadrelated complications requiring surgical revision.

Another potential limitation of the first S-ICD system was the battery longevity of about 5 years, which is shorter than T-ICDs, meaning more replacements and potential complications. ${ }^{64}$ However, the new S-ICD generation (EMBLEM MRI model A219, Boston Scientific, USA) is an advanced MRI conditional technology with a prolonged battery life of up to 7 years
(http://www.bostonscientific.com/en-US/products/defibrillators/emblem-s-icd-system/device-overview/specifications.html). Finally, the higher cost of the S-ICD compared with T-ICD could be a limitation in clinical practice. However, despite the existing significant difference in unit cost of the S-ICD, overall S-ICD costs may be mitigated vs. T-ICDs over a longer follow-up period. ${ }^{65}$

\section{Conclusion}

The S-ICD represents an important advance in clinical electrophysiology, offering life-saving defibrillation while limiting lead-related complications. Technical refinements including size reduction, increased battery longevity, and improved T-wave detection will enhance its clinical utility. The availability of a defibrillator system associated with fewer complications than traditional $\mathrm{T}$ ICD may expand the proportion of patients at risk of SCD to whom ICD therapy confers a net clinical benefit. Specifically, it may lower the threshold for ICD implantation in patients with cardiomyopathies who have a long quality-adjusted life expectancy but are also particularly prone to the risk of complications of traditional ICD (and dangerous transvenous lead-extraction procedures).

\section{Conflicts of interest}

There are no conflicts of interest.

\section{References}

1 Maron BJ, Towbin JA, Thiene G, et al. Contemporary definitions and classification of the cardiomyopathies: an American Heart Association Scientific Statement from the Council on Clinical Cardiology, Heart Failure and Transplantation Committee; Quality of Care and Outcomes Research and Functional Genomics and Translational Biology Interdisciplinary Working Groups; and Council on Epidemiology and Prevention. Circulation 2006; 113:1807-1816. 
2 Nichol G, Sayre MR, Guerra F, et al. Defibrillation for ventricular fibrillation: a shocking update. J Am Coll Cardiol 2017; 70:1496-1509.

3 Al-Khatib SM, Friedman P, Ellenbogen KA. Defibrillators: selecting the right device for the right patient. Circulation 2016; 134:1390-1404.

4 Lewis GF, Gold MR. Safety and efficacy of the subcutaneous implantable defibrillator. J Am Coll Cardiol 2016; 67:445-454.

5 McLeod CJ, Boersma L, Okamura H, et al. The subcutaneous implantable cardioverter defibrillator: state-of-the-art review. Eur Heart J 2017; 38:247-225.

6 Hawkins NM, Grubisic M, Andrade JG, et al. Long-term complications, reoperations and survival following cardioverter-defibrillator implant. Heart 2017; 104:237-243.

7 Al-Khatib SM, Stevenson WG, Ackerman MJ, et al. 2017 AHA/ACC/HRS Guideline for management of patients with ventricular arrhythmias and the prevention of sudden cardiac death: a report of the American College of Cardiology/American Heart Association Task Force on Clinical Practice Guidelines and the Heart Rhythm Society. J Am Coll Cardiol 2017; pii: S0735-1097 (17)41306-4.

8 Kirkfeldt RE, Johansen JB, Nohr EA, et al. Complications after cardiac implantable electronic device implantations: an analysis of a complete, nationwide cohort in Denmark. Eur Heart J 2014; 35:1186-1194.

9 Tarakji KG, Wazni OM, Wilkoff BL, et al. Risk factors for 1-year mortality among patients with cardiac implantable electronic device infection undergoing transvenous lead extraction: the impact of the infection type and the presence of vegetation on survival. Europace 2014; 16:490-495.

10 Olde Nordkamp LR, Postema PG, Knops RE, et al. Implantable cardioverter-defibrillator harm in young patients with inherited arrhythmia syndromes: a systematic review and meta-analysis of inappropriate shocks and complications. Heart Rhythm 2013; 13:443-454.

11 Migliore F, Silvano M, Zorzi A, et al. Implantable cardioverter defibrillator therapy In young patients with cardiomyopathies and channelopathies: a single Italian centre experience. J Cardiovasc Med 2016; 17:485-493.

12 Boersma L, Barr C, Knops R, et al., EFFORTLESS Investigator Group. Implant and midterm outcomes of the Subcutaneous Implantable Cardioverter-Defibrillator Registry: the EFFORTLESS Study. J Am Coll Cardiol 2017; 70:830-841.

13 Knops RE, Olde Nordkamp LR, de Groot JR, et al. Two-incision technique for implantation of the subcutaneous implantable cardioverter defibrillator. Heart Rhythm 2013; 10:1240-1243.

14 Migliore F, Bottio T, lliceto S, et al. Submuscular approach for subcutaneous implantable cardioverter defibrillator: a potential alternative technique. J Cardiovasc Electrophysiol 2015; 26:905.

15 Ferrari $P$, Giofrè $F$, De Filippo $P$. Intermuscular pocket for subcutaneous implantable cardioverter defibrillator: single-center experience. J Arrhythm 2016; 32:223-226.

16 Migliore F, Allocca G, Calzolari V, et al. Intermuscular two-incision technique for subcutaneous implantable cardioverter defibrillator implantation: results from a multicenter registry. Pacing Clin Electrophysiol 2017; 40:278-285.

17 Friedman DJ, Parzynski CS, Varosy PD, et al. Trends and in-hospital outcomes associated with adoption of the subcutaneous implantable cardioverter defibrillator in the United States. JAMA Cardiol 2016; 1:900-911.

18 Schinkel AF, Vriesendorp PA, Sijbrands EJ, et al. Outcome and complications after implantable cardioverter defibrillator therapy in hypertrophic cardiomyopathy: systematic review and meta-analysis. Circ Heart Fail 2012; 5:552-559.

19 Rudic B, Tulumen E, Berlin V, et al. Low prevalence of inappropriate shocks in patients with inherited arrhythmia syndromes with the subcutaneous implantable defibrillator single center experience and long-term follow-up. J Am Heart Assoc 2017; 6: pii: e006265.

20 Priori S, Blomström-Lundqvist C, Mazzanti A, et al., ESC Scientific Document Group. ESC Guidelines for the management of patients with ventricular arrhythmias and the prevention of sudden cardiac death: the task force for the management of patients with ventricular arrhythmias and the prevention of sudden cardiac death of the European Society of Cardiology. Eur Heart J 2015; 36:2793-2867.

21 Authors/Task Force members, Elliott PM, Anastasakis MA, et al. 2014 ESC Guidelines on diagnosis and management of hypertrophic cardiomyopathy: The Task Force for the diagnosis and management of hypertrophic cardiomyopathy of the European Society of Cardiology. Eur Heart J 2014; 35:2733-2779

22 Jarman JW, Todd DM. United Kingdom national experience of entirely subcutaneous implantable cardioverter-defibrillator technology: important lessons to learn. Europace 2013; 15:1158-1165.

23 Maurizi N, Olivotto I, Olde Nordkamp LR, et al. Prevalence of subcutaneous implantable cardioverter-defibrillator candidacy based on template ECG screening in patients with hypertrophic cardiomyopathy. Heart Rhythm 2016; 13:457-463.
24 Francia P, Adduci C, Palano F, et al. Eligibility for the subcutaneous implantable cardioverter-defibrillator in patients with hypertrophic cardiomyopathy. J Cardiovasc Electrophysiol 2015; 26:893-899.

25 Srinivasan NT, Patel KH, Qamar K, et al. Disease severity and exercise testing reduce subcutaneous implantable cardioverter-defibrillator left sternal ECG screening success in hypertrophic cardiomyopathy. Circ Arrhythm Electrophysiol 2017; 10: pii: e004801.

26 Maurizi N, Tanini I, Olivotto I, et al. Effectiveness of subcutaneous implantable cardioverter-defibrillator testing in patients with hypertrophic cardiomyopathy. Int J Cardiol 2017; 231:115-119.

27 Weinstock J, Bader YH, Maron MS, et al. Subcutaneous implantable cardioverter defibrillator in patients with hypertrophic cardiomyopathy: an initial experience. J Am Heart Assoc 2016; 5: pii: e002488.

28 Lambiase PD, Gold MR, Hood M, et al. Evaluation of subcutaneous ICD early performance in hypertrophic cardiomyopathy from the pooled EFFORTLESS and IDE cohorts. Heart Rhythm 2016; 13:1066-1074.

29 Moss AJ, Zareba W, Hall WJ, et al., Multicenter Automatic Defibrillator Implantation Trial II Investigators. Prophylactic Implantation of a defibrillator in patients with myocardial infarction and reduced ejection fraction. N Eng $J$ Med 2002; 346:877-883.

30 Moss AJ, Schuger C, Beck CA, et al., MADIT-RIT Trial Investigators. Reduction in inappropriate therapy and mortality through ICD programming. N Engl J Med 2012; 24:2275-2283.

31 Sideris S, Archontakis S, Gatzoulis KA, et al. The subcutaneous ICD as an alternative to the conventional ICD system: initial experience in Greece and a review of the literature. Hellenic $J$ Cardiol 2017; 58:4-16.

32 Ruwald AC, Schuger C, Moss AJ, et al. Mortality reduction in relation to implantable cardioverter defibrillator programming in the Multicenter Automatic Defibrillator Implantation Trial-Reduce Inappropriate Therapy (MADIT-RIT). Circ Arrhythm Electrophysiol 2014; 7: $785-792$.

33 Brouwer TF, Yilmaz D, Lindeboom R, et al. Long-term clinical outcomes of subcutaneous versus transvenous implantable defibrillator therapy. J Am Coll Cardiol 2016; 19:2047-2055.

34 Gold MR, Knops R, Burke MC, et al. The design of the understanding outcomes with the S-ICD in primary prevention patients with low EF study (UNTOUCHED). Pacing Clin Electrophysiol 2017; 40:1-8.

35 Corrado D, Wichter T, Link MS, et al. Treatment of arrhythmogenic right ventricular cardiomyopathy/dysplasia: an international Task Force Consensus Statement. Circulation 2015; 132:441-453.

36 Link MS, Laidlaw D, Polonsky B, et al. Ventricular arrhythmias in the North American multidisciplinary study of ARVC: predictors, characteristics and treatment. J Am Coll Cardiol 2014; 64:119-125.

37 Corrado D, Link MS, Calkins H. Arrhythmogenic right ventricular cardiomyopathy. N Engl J Med 2017; 376:1489-1490.

38 Corrado D, Basso C, Judge DP. Arrhythmogenic cardiomyopathy. Circ Res 2017; 121:784-802.

39 Allocca A, Sitta N, Turiano G. Inappropriate shocks by subcutaneous defibrillator in a patient with arrhythmogenic right ventricular cardiomyopathy: problem fixed. Europace 2015; 17:1067.

40 Migliore F, Bertaglia E, Zorzi A, et al. Subcutaneous implantable cardioverter defibrillator and arrhythmogenic cardiomyopathy the importance of repeat ECG screening during exercise test. JACC Clinical Electrophysiol 2017; 3:785-786.

41 Migliore F, Zorzi A, Michieli P, et al. Prevalence of cardiomyopathy in Italian asymptomatic children with electrocardiographic T-wave inversion at preparticipation screening. Circulation 2012; 125:529-538.

42 Curcio A, Santarpia G, Indolfi I. The Brugada syndrome - from gene to therapy. Circ J 2017; 3:290-297.

43 Antzelevitch C, Yan GX, Ackerman MJ, Borggrefe M, et al. J-Wave syndromes expert consensus conference report: Emerging concepts and gaps in knowledge. Europace 2017; 19:665-694.

44 Priori SG, Gasparini M, Napolitano C, et al. Risk stratification in Brugada syndrome: results of the PRELUDE (PRogrammed ELectrical stimUlation preDictive valuE) registry. J Am Coll Cardiol 2012; 59:37-45.

45 Curcio A, Mazzanti A, Bloise R, et al. Clinical presentation and outcome of Brugada syndrome diagnosed with the new 2013 criteria. J Cardiovasc Electrophysiol 2016; 27:937-943.

46 Giustetto C, Cerrato N, Gribaudo E, et al. Atrial fibrillation in a large population with Brugada electrocardiographic pattern: prevalence, management, and correlation with prognosis. Heart Rhythm 2014 11:259-265

47 Veltmann $\mathrm{C}, \mathrm{H}$. Barajas-Martinez $\mathrm{H}$, Wolpert $\mathrm{C}$, et al. Further insights in the most common SCN5A mutation causing overlapping phenotype of long QT syndrome, Brugada syndrome, and conduction defect. J Am Heart Assoc 2016; 5: pii: e003379. 
48 Migliore F, Testolina M, Zorzi A, et al. First-degree atrioventricular block on basal electrocardiogram predicts future arrhythmic events in patients with Brugada syndrome: a long-term follow-up study from the Veneto region of Northeastern Italy. Eurospace 2018; [Epub ahead of print].

49 Rodríguez-Mañero M, Sacher F, de Asmundis C, et al. Monomorphic ventricular tachycardia in patients with Brugada syndrome: a multicenter retrospective study. Heart Rhythm 2016; 13:669-682.

50 Ishibashi K, Noda T, Kamakura T, et al. Importance of exercise testing shortly after subcutaneous implantable cardioverter-defibrillator implantation in patients with Brugada syndrome - the first case of associated inappropriate shock in Japan. J Arrhythm 2017; 33:156-158.

51 Kamakura T, Sato T, Wada M, et al. T-wave oversensing during drug challenge test after subcutaneous implantable cardioverter-defibrillator implantation in a patient with Brugada syndrome. Heart Rhythm Case Rep 2016; 13:391-394.

52 Brisben AJ, Burke MC, Knight BP, et al. A new algorithm to reduce inappropriate therapy in the S-ICD system. J Cardiovasc Electrophysiol 2015; 26:417-423.

53 Gold MR, Theuns DA, Knight BP, et al. Head-to-head comparison of arrhythmia discrimination performance of subcutaneous and transvenous ICD arrhythmia detection algorithms: the START study. J Cardiovasc Electrophysiol 2012; 23:359-366.

54 Randles DA, Hawkins NM, Shaw M, et al. How many patients fulfil the surface electrocardiogram criteria for subcutaneous implantable cardioverter-defibrillator implantation? Europace 2014; 16:1015-1021.

55 Campbell M, Moore JP, Sreeram N, et al. Predictors of electrocardiographic screening failure for the subcutaneous implantable cardioverter-defibrillator in children: a prospective multicenter study. Heart Rhythm 2018; 15:703-707.

56 Olde Nordkamp LR, Warnaars JLF, Kooiman KM, et al. Which patients are not suitable for a subcutaneous ICD: incidence and predictors of failed QRS-T-wave morphology screening. J Cardiovasc Electrophysio/ 2014; 25:494-499.
57 Olde Nordkamp LR, Dabiri Abkenari L, Boersma LV, et al. The entirely subcutaneous implantable cardioverter-defibrillator: initial clinical experience in a large Dutch cohort. J Am Coll Cardiol 2012; 60:19331939.

58 Groh CA, Sharma S, Pelchovitz DJ, et al. Use of an electrocardiographic screening tool to determine candidacy for a subcutaneous implantable cardioverter-defibrillator. Heart Rhythm 2014; 1:1361-1366.

59 Conte G, Kawabata M, de Asmundis $\mathrm{C}$, et al. High rate of subcutaneous implantable cardioverter-defibrillator sensing screening failure in patients with Brugada syndrome: a comparison with other inherited primary arrhythmia syndromes. Europace 2018; 20:1188-1193.

60 Rudic B, Tülümen E, Berlin V, et al. Low prevalence of inappropriate shocks in patients with inherited arrhythmia syndromes with the subcutaneous implantable defibrillator single center experience and long-term follow-up. $J$ Am Heart Assoc 2017; 6: pii: e006265.

61 Ziacchi M, Corzani A, Diemberger I, et al. Electrocardiographic eligibility for subcutaneous implantable cardioverter defibrillator: evaluation during bicycle exercise. Heart Lung Circ 2016; 25:476-483.

62 Boriani G, Artale P, Biffi M, et al. Outcome of cardioverter-defibrillator implant in patients with arrhythmogenic right ventricular cardiomyopathy. Heart Vessels 2007; 22:184-192.

63 Santini L, Pappalardo A, Schirripa V, et al. A new tool to improve detection of supraventricular arrhythmias in subcutaneous implantable cardioverterdefibrillators. Heart Rhythm Case Rep 2017; 3:286-288.

64 Borleffs CJ, Thijssen J, de Bie MK, et al. Recurrent implantable cardioverterdefibrillator replacement is associated with an increasing risk of pocketrelated complications. Pacing Clin Electrophysiol 2010; 33:1013-1019.

65 Honarbakhsh S, Providencia R, Srinivasan N, et al. A propensity matched case-control study comparing efficacy, safety and costs of the subcutaneous vs. transvenous implantable cardioverter defibrillator. Int J Cardiol 2017; 228:280-285. 